\title{
ORIGINAL
}

\section{High intensity zone in lumbar spine and its correlation with disc degeneration}

\author{
Subash C. Jha, Yoichiro Takata, Mitsunobu Abe, Kazuta Yamashita, Fumitake Tezuka, Toshinori Sakai, \\ Kosaku Higashino, Akihiro Nagamachi, and Koichi Sairyo \\ Department of Orthopedics, Tokushima University, Tokushima, Japan
}

\begin{abstract}
Purpose ; To investigate the prevalence, clinical significance of high intensity zone (HIZ), and associated disc degeneration. Materials and methods; We undertook retrospective analysis of 228 patients, aged from 15 to 55 years, who had undergone magnetic resonance (MR) imaging of lumbar spine in outpatient clinic from 2013 to 2014. HIZ was defined as a presence of high intensity signal in the annulus on T2-weighted images. All HIZ positive discs were classified according to Pfirrmann grading system for presence of disc degeneration. Results ; Forty - three patients (18.8\%) with 53 intervertebral discs demonstrated HIZ. There was no significant predominance about age and gender for the presence of HIZ. HIZ occurred mainly at L4-5 (43.3\%) and L5-S1 (30.2\%) levels, infrequently at L3-4 (17\%) and rarely at upper levels. The prevalence of disc degenerations with Pfirrmann grade 3, 4, and 5 were significantly higher in HIZ positive patients than in randomly selected 35 HIZ negative patients $(64 \%$ vs. $46 \%$, respectively). Conclusion ; In this study we identified that the presence of HIZ was directly proportional to increased incidence of disc degeneration in remaining lumbar discs. The prevalence of significant degeneration was significantly higher in HIZ positive patient at lower three lumbar levels. J. Med.Invest. 64 : 39 - 42, February, 2017
\end{abstract}

Keywords : High intensity zone, lumbar disc degeneration, magnetic resonance image

\section{INTRODUCTION}

High intensity zone (HIZ) is defined as a significantly high intensity signal on $\mathrm{T} 2$-weighted magnetic resonance (MR) images located in the substance of annulus fibrosus clearly dissociated from signal of nucleus pulposus. It was first described by Aprill and Bogduk in 1992 with prevalence of $28 \%$ in symptomatic patients (1). They reported sensitivity, specificity, and positive predictive value of $71 \%, 89 \%$, and $86 \%$ respectively. Initially, it was only described at posterior annulus but now posterolateral or even circumferential lesions are also included in the definition (2-4). It is described as a sign of painful internal disc disruption on MR images, thereby limiting the role of provocative discography for diagnosis of discogenic low back pain (DLBP). Various study have been done regarding its significance in clinical practice, many of them have demonstrated it as a significant MR finding, while few are against its role in diagnosing DLBP.

Now many authors believe that true HIZ has to be differentiated from low/medium intensity zone, depending on signal intensity, thereby improving the diagnostic value of the sign (4-7). The prevalence of HIZ varies from $28-59 \%$ in symptomatic individuals $(1,4,6)$. In this study we investigated HIZ in terms of prevalence, clinical significance and associated disc degeneration in $228 \mathrm{pa}-$ tients aged between $15-55$ years, who visited our out-patient clinic, to whom lumbar MR study was done irrespective of their complaint.

Received for publication July 6, 2016 ; accepted August 29, 2016.

Address correspondence and reprint requests to Yoichiro Takata, $\mathrm{MD}, \mathrm{PhD}$, Department of Orthopedics, Tokushima University 3-18-15 Kuramoto-cho, Tokushima 770-8503, Japan and Fax : +81-88-0-6330178 .

\section{SUBJECTS AND METHODS}

An institutional review board exemption was obtained for the review of imaging studies, the indications for the studies.

We undertook retrospective analysis of patients who had undergone MR imaging of lumbar spine irrespective of their clinical history, from January 2013 to December 2014. From 1039 consecutive patients, 228 patients aged 15-55 years old were sort out and reviewed for the presence of HIZ. All the MR images were conducted on Signa Excite HD 1.5 tesla MR system (GE Yokogawa Medical System, Tokyo, Japan) with patients in the supine position. The examination was done by sagittal $\mathrm{T} 2$-weighted fast spin echo (TR: 3000 to $4500 \mathrm{~ms}$, TE : 100 to $130 \mathrm{~ms}$, section thickness $4 \mathrm{~mm}$, intersection gap $1 \mathrm{~mm}$ ), axial T2-weighted fast spin echo (TR: 3500 to $5000 \mathrm{~ms}$, TE : 85 to $105 \mathrm{~ms}$, section thickness $4 \mathrm{~mm}$, intersection gap $1 \mathrm{~mm}$ ). The HIZ was identified on sagittal and/or axial slice of T2-weighted images (Figure 1). Presence of significantly high intensity signal in annulus, which was as intense as cerebrospinal fluid signal located adjacent to the disc, was defined as HIZ. We compared number of patients, average age, gender differences, age distribution between HIZ positive groups and HIZ negative groups. All HIZ positive discs were classified according to Pfirrmann grading system for presence of disc degeneration. Prevalences of disc degeneration more than equal to Pfirrmann grade 3 , in each lumbar intervertebral disc (IVD) were compared between HIZ positive patients and randomly selected $35 \mathrm{HIZ}$ negative patients. Statistical analysis was performed with SPSS Statistics version 22.0 (IBM Corp. Armonk, NY).

\section{RESULTS}

Forty three patients (18.9\%) demonstrated at least one HIZ positive disc. There was no significant difference in gender predominance. The prevalence was almost equal among the young 
A)

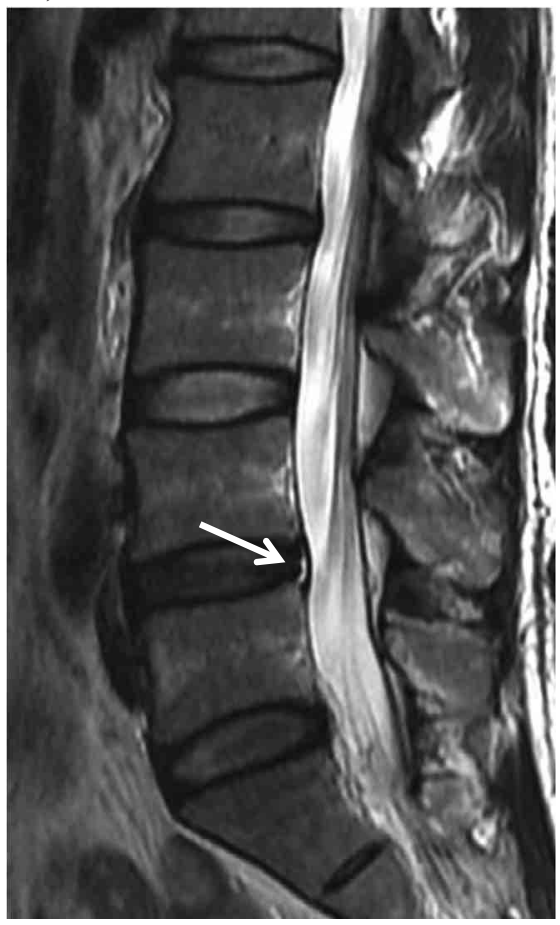

B)

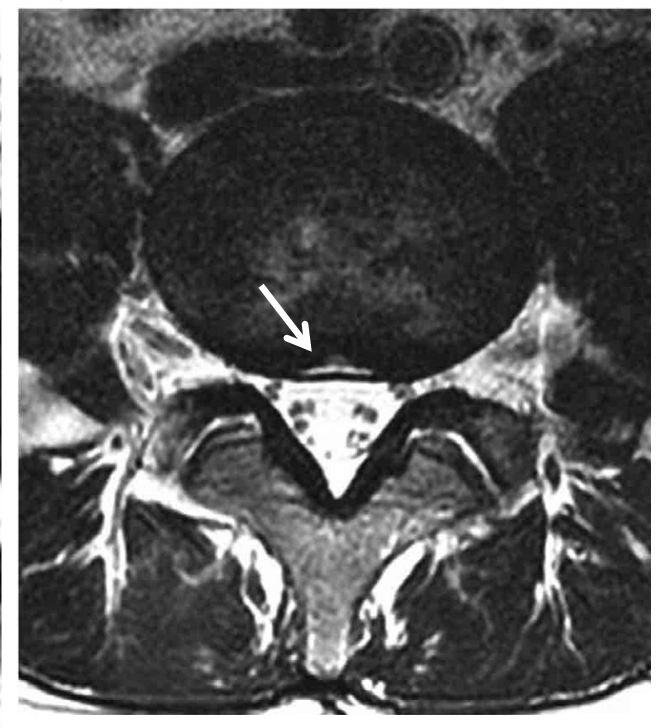

Figure 1 : Sagittal (A) and axial (B) views of T2-weighted images showing high-intensity zone in the posterior annulus at L4-5 level with Pfirrmann grade 3 disc degeneration (arrows).

age (15-40 years) and middle age (41-55 years) (Table 1$)$.

Out of 43 HIZ positive patients, 34 (79.1\%) had single level HIZ positive disc and 9 (20.9\%) had multilevel HIZ positive discs. Among 9 patients with multilevel HIZ, 8 had in 2 IVD levels and 1 had it in 3 IVD levels. In total, 53 IVDs were HIZ positive. HIZs were found mainly at L4-5 and L5-S1 levels, infrequently at L3-4 and rare at L2-3 and L1-2. Majority of HIZ (87\%) were identified at posterior annulus, but a significant numbers were also present at posterolateral annulus (13\%), which were identified on para-sagittal or axial slice of MR images (Figure 2).

When classified according to Pfirrmann grading, 38 (71.7\%) discs had grade 4 degeneration while $9(17 \%)$ and $6(11.3 \%)$ discs had grade 3 and grade 5 degeneration, respectively. We compared the prevalence of disc degeneration in HIZ positive patients with that of randomly selected $35 \mathrm{HIZ}$ negative patients. Total 215 IVDs in $43 \mathrm{HIZ}$ positive patients and 175 IVDs in 35 HIZ negative patients were examined for the disc degeneration and classified according to Pfirrmann grading system. Pfirrmann grade 3,4 and 5 were defined as significant degeneration. In total, 142 (64\%) IVDs in HIZ positive patients had significant degeneration, while only 82 (46.4\%) IVD were identified with significant degeneration in HIZ negative patients (Table 2). The number of degenerated disc was significantly higher in lower three lumbar IVDs (L3-4, L4-5, and L5-S1) in HIZ positive group when compared with HIZ negative group.

\section{DISCUSSION}

HIZ was first identified as a pain indicator in 1992 (1). Various studies demonstrated its clinical relevance in diagnosing DLBP $(3,4,8)$. But, the high prevalence of HIZ in asymptomatic population brought its significance under controversy $(6,9,10)$. Recently, more precise diagnosis of HIZ considering significant high intensity signal and location has made it an established indicator of painful internal disc disruption.

HIZ is believed to be the detached nucleus pulposus trapped between the lamellae of torn annulus fibrosus, following secondary inflammation, causing characteristic signal on MR imaging. Presence of granulation tissue or neovascularization induced by inflammation was demonstrated by Ross (11). Most of the nerve supply of the intervertebral disc is limited to periphery of the annulus fibrosus (12-14). Hence, the annular tear extending to the inner third

Table 1. Prevalence and distribution of HIZ according to gender and age.

\begin{tabular}{|c|c|c|c|}
\hline & HIZ $(+)$ & HIZ (-) & \\
\hline Age (average \pm SD) & $\mathrm{n}=43$ & $\mathrm{n}=185$ & \\
\hline male $:$ female & $39.0 \pm 11$ & $39.3 \pm 13$ & n.s. \\
\hline young age (15-40) & $24: 19$ & $85: 100$ & n.s. \\
\hline middle age (41-55) & 22 & 83 & n.s. \\
\hline
\end{tabular}

HIZ : high intensity zone, n.s. : not significant 


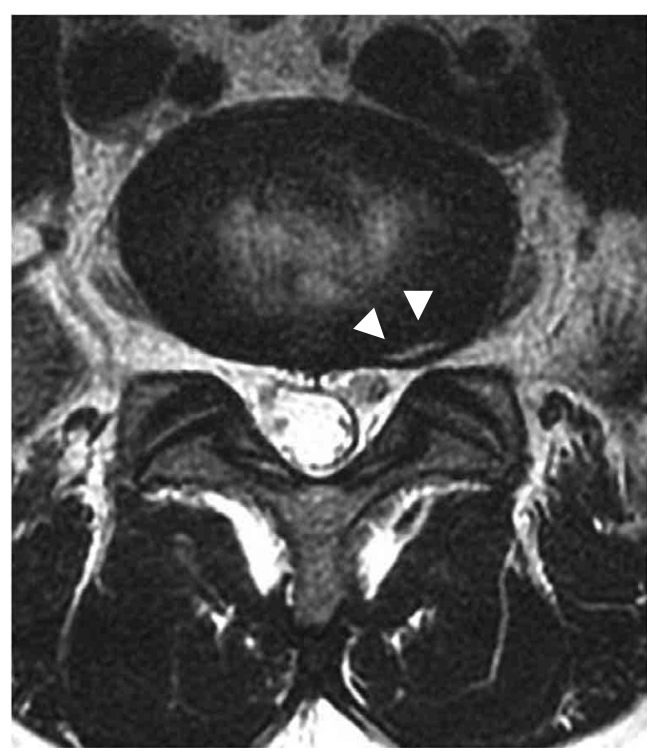

Figure 2 : Axial view of T2-weighted MR image showing high-intensity zone in left posterolateral annulus at L5-S level (arrow heads).

of the annulus fibrosus are asymptomatic while tear extending to peripheral third produces pain (15). Biomechanical mediators and pro-inflammatory cytokines are more important than mechanical compression in the pathogenesis of pain production (16).

Originally, HIZ was described in T2-weighted sequence but with growing controversies diagnosing true HIZ has become a challenge to the clinicians. Liu et al. (4) defined true HIZ as a lesion with signal intensity at least as bright as $50 \%$ of the CSF signal intensity. The quantitative measurement was performed by Picture Archiving and Communication System, with embedded region of interest and grayscale histogram software. They recommended this method to increase measurement precision and reduce error in identification of true HIZ.

Posterior annulus fibrosus is structurally weak, and exposed to high stress concentration (17). Hence, maximum number of HIZ occurs posteriorly. But, significant number of HIZ are identified at posterolateral annulus or even circumferentially. Rankine et al. (9) in the study of 156 cases, reported $77 \%$ of HIZ at posterior annulus while $22 \%$ at posterolateral annulus. Previous studies have already demonstrated that most of the HIZ are identified at lower lumbar level mainly at L4-5 and L5-S levels $(2,3,18)$.

Similar to previous studies, we also experienced severe degree of disc degeneration in all HIZ positive discs. Lam et al. (8) has demonstrated that there was significant correlation between abnormal disc morphology and presence of HIZ. No IVD that exhibited HIZ had a normal level of disc hydration as seen on T2-weighted MR images. The sensitivity, specificity and positive predictive value of HIZ positive discs showing disc dehydration were $68 \%, 100 \%$, and $100 \%$ respectively. Similarly, in our study all the HIZ positive discs were invariably dehydrated. In our study we also tried to evaluate the relationship of HIZ positive patient with presence of disc degeneration, according to Pfirrmann grading on MR imaging at other lumbar IVD. We compared the results with HIZ negative patients. The prevalence of significant degeneration was significantly higher in HIZ positive patient at lower three lumbar levels. It signifies that HIZ positive patient are more susceptible or vulnerable for degeneration of adjacent level discs compared to HIZ negative patient.

The prevalence of HIZ in our study is less compared to other reports, as we have included the entire patient irrespective of their complaint or history. Posterior location of HIZ was the most common but there was significant number of HIZ identified at posterolateral annulus. In contrast to previous studies, we evaluated the relation of disc degeneration in other lumbar IVD in HIZ positive patients compared to HIZ negative patients. In this study we identified that the presence of HIZ even in one IVD is directly proportional to increased incidence of disc degeneration in remaining lumbar IVD, mainly on lower lumbar levels.

\section{CONFLICT OF INTEREST}

The authors declare that they have no conflict of interest.

\section{REFERENCES}

1. Aprill C, Bogduk N : High-intensity zone : a diagnostic sign of painful lumbar disc on magnetic resonance imaging. Br J Radiol 65 : 361-369, 1992

2. Schellhas KP, Pollei SR, Gundry CR, Heithoff KB : Lumbar disc high-intensity zone. Correlation of magnetic resonance imaging and discography. Spine $21: 79-86,1996$

3. Wang Z-X, Hu Y-G : High-intensity zone (HIZ) of lumbar intervertebral disc on $\mathrm{T} 2$-weighted magnetic resonance images : spatial distribution, and correlation of distribution with low back pain (LBP). Eur Spine J 21 : 1311-1315, 2012

4. Liu C, Cai H-X, Zhang J-F, Ma J-J, Lu Y-J, Fan S-W : Quantitative estimation of the high-intensity zone in the lumbar spine : comparison between the symptomatic and asymptomatic population. Spine J $14: 391-396,2014$

5. Ito M, Incorvaia KM, Yu SF, Fredrickson BE, Yuan HA, Rosenbaum AE : Predictive signs of discogenic lumbar pain on magnetic resonance imaging with discography correlation. Spine 23 : 1252-1260, 1998

6. Carragee EJ, Paragioudakis SJ, Khurana S : 2000 Volvo Award winner in clinical studies : Lumbar high-intensity zone and

Table2. Prevalence of discs degeneration at each lumbar IVD, compared between HIZ negative and HIZ positive patients.

\begin{tabular}{|c|c|c|c|c|c|c|c|}
\hline & HIZ (+) group & & & HIZ (-) group & & & \\
\hline & DD+ & DD- & $\%$ DD + & DD + & DD- & $\% D D+$ & \\
\hline Overall & 142 & 73 & $66.0 \%$ & 82 & 93 & $46.9 \%$ & $\mathrm{p}=0.0001$ \\
\hline L1-2 & 15 & 28 & $34.9 \%$ & 12 & 23 & $34.3 \%$ & $\mathrm{p}=0.96$ \\
\hline L2-3 & 19 & 24 & $44.2 \%$ & 11 & 24 & $31.4 \%$ & $\mathrm{p}=0.25$ \\
\hline L3-4 & 29 & 14 & $67.4 \%$ & 15 & 23 & $34.3 \%$ & $\mathrm{p}=0.003$ \\
\hline L4-5 & 42 & 1 & $97.7 \%$ & 24 & 11 & $68.6 \%$ & $\mathrm{p}=0.0004$ \\
\hline L5-S1 & 37 & 6 & $86.0 \%$ & 23 & 12 & $65.7 \%$ & $\mathrm{p}=0.034$ \\
\hline
\end{tabular}

HIZ : high intensity zone, DD : disc degeneration 
discography in subjects without low back problems. Spine 25 : 2987-2992, 2000

7. O'Neill C, Kurgansky M, Kaiser J, Lau W : Accuracy of MRI for diagnosis of discogenic pain. Pain Physician 11:311-326, 2008

8. Lam KS, Carlin D, Mulholland RC : Lumbar disc high-intensity zone : the value and significance of provocative discography in the determination of the discogenic pain source. Eur Spine J 9 : 36-41, 2000

9. Rankine JJ, Gill KP, Hutchinson CE, Ross ER, Williamson JB : The clinical significance of the high-intensity zone on lumbar spine magnetic resonance imaging. Spine 24 : 1913-1920, 1999

10. Mitra D, Cassar-Pullicino VN, McCall IW : Longitudinal study of high intensity zones on MR of lumbar intervertebral discs. Clin Radiol 59 : 1002-1008, 2004

11. Ross JS, Modic MT, Masaryk TJ : Tears of the anulus fibrosus : assessment with Gd-DTPA-enhanced MR imaging. Am J Roentgenol $154:$ 159-162, 1990

12. Bogduk N, Tynan W, Wilson AS : The nerve supply to the human lumbar intervertebral discs. J Anat 132 : 39-56, 1981

13. Palmgren T, Grönblad M, Virri J, Kääpä E, Karaharju E : An immunohistochemical study of nerve structures in the anulus fibrosus of human normal lumbar intervertebral discs. Spine $24: 2075-2079,1999$

14. García-Cosamalón J, del Valle ME, Calavia MG, García-Suárez O, López-Muñiz A, Otero JV, José A : Intervertebral disc, sensory nerves and neurotrophins : who is who in discogenic pain? J Anat $217: 1-15,2010$

15. Sachs BL, Vanharanta H, Spivey MA, Guyer RD, Videman T, Rashbaum RF, Johnson RG, Hochschuler SH, Mooney V : Dallas discogram description. A new classification of CT/discography in low-back disorders. Spine $12: 287-294,1987$

16. Burke JG, Watson RWG, McCormack D, Dowling FE, Walsh MG, Fitzpatrick JM : Intervertebral discs which cause low back pain secrete high levels of proinflammatory mediators. J Bone Joint Surg Br 84 : 196-201, 2002

17. Wang $Z$-X, Hu Y-G : [Clinical investigation of high-intensity zone in anterior annulus fibrosus of lumbar disc : compared with high-intensity zone in posterior annulus fibrosus]. Zhonghua Wai Ke Za Zhi 47 : 689-692, 2009

18. Saifuddin A, Braithwaite I, White J, Taylor BA, Renton P : The value of lumbar spine magnetic resonance imaging in the demonstration of anular tears. Spine $23: 453-457,1998$ 